\title{
Services Provisioning on Mobile Devices via Bluetooth in a Localized Setting Using a Proposed System - BTServ
}

\section{Oludele Awodele, Victor Dibia, Oghenerukevwe Onoruvie, and Sharon Okoruwa \\ Babcock University, Department of Computer Science and Mathematics, Ilishan-Remo, Ogun State, Nigeria}

\author{
delealways@yahoo.com, chuvidi2003@yahoo.com, \\ rukkivie@yahoo.com, sharonville2003@yahoo.com,
}

\begin{abstract}
Conventionally, enterprise services that can be utilized by mobile devices such as E - mail, instant messaging, data transfer, news and event notification have been typically achieved using GPRS (General Packet Radio Service) connections to access internet servers that provide these services. Thus, even in a local area, disadvantages such as full dependence on GPRS service providers, incurred GPRS costs and slow data/communication rates constitute formidable drawbacks. This paper describes a system /Architecture (BTServ) in which these same services can be supplied via Bluetooth, with notable benefits such as high data rates, minimal dependence on external (GPRS) networks, higher security (internet attacks are totally avoided) and reduced cost, in a localized setting such as a corporate house.
\end{abstract}

Keywords: Bluetooth LAN, Bluetooth Programming, JSR 82 API, Mobile computing, J2ME MIDlet

\section{Introduction}

Mobile computing is, basically, the use of heterogeneous mobile devices (such as mobile phones and Personal Digital Assistants, Notebook computers ) and a variety of embedded computer systems (such as smart cards and vehicle onboard computers) in achieving computing needs "on the fly" ("Mobile Computing", 2007). Services for mobile devices are the back bone of mobile computing which is fast becoming a major means of information dissemination, in corporate organizations.

Currently, the internet is the major framework through which enterprise services are provided to mobile devices, in which devices con-

Material published as part of this publication, either on-line or in print, is copyrighted by the Informing Science Institute. Permission to make digital or paper copy of part or all of these works for personal or classroom use is granted without fee provided that the copies are not made or distributed for profit or commercial advantage AND that copies 1) bear this notice in full and 2) give the full citation on the first page. It is permissible to abstract these works so long as credit is given. To copy in all other cases or to republish or to post on a server or to redistribute to lists requires specific permission and payment of a fee. Contact Publisher@InformingScience.org to request redistribution permission. nect to the servers via GPRS. Though this method offers large area of coverage, it also has characteristic features such as:

- $\quad$ Dependence on GPRS Network from GSM (Global System for Mobile Telecommunications) Provider,

- $\quad$ GPRS Costs, 
- Low Data Transfer Rate,

- $\quad$ Security Risks associated with internet transactions.

These features reduce the efficiency and economy achieved, especially when considering a localized environment such as a large office block, where intranet services provided are intended for "in house" consumption. However, these services can also be made available via Bluetooth. Why connect through GPRS, incurring costs, to check your intranet mail or send instant messages to a colleague or upload sensitive data to your boss, when you could handle that effectively via Bluetooth connection on you mobile device, provided the necessary system is available? Bluetooth LAN connections, which are fairly common today, are used for interconnecting small devices such as printers, PDA's (Personal Digital Assistants) and notebook computers simply for data sharing. This paper explores the angle of utilizing this same Bluetooth links for providing enterprise intranet services to mobile devices (Bluetooth capable Smart Phones and PDA's) in an efficient manner.

\section{GPRS or Bluetooth?}

This section discusses characteristics of the GPRS and Bluetooth standards for data communication, relevant to services provisioning on mobile devices.

\section{Overview of GPRS}

According VOCAL Technologies (2001), "GPRS or General Packet Radio Service is a packet switched technology, based on GSM. The radio and network resources of GPRS are only accessed when data actually needs to be transmitted between the GPRS mobile user and the GPRS network. This data is divided into packets and is then transferred via the radio and core GPRS network. Between alternating transmissions, no GPRS network resources need to be allocated. GPRS offers up to $115 \mathrm{kbps}$, depending on the network availability, channel coding scheme and terminal capability. This increase in speed in GPRS with respect to GSM is achieved by using more than one timeslot of the TDMA frame." "TDMA (Time Division Multiple Access) is a technology used in digital cellular telephone communication that divides each cellular channel into three time slots in order to increase the amount of data that can be carried "(TDMA, 2006). "Due to the packet switched characteristics of GPRS the allocation of the available timeslots may vary from one instant to the next (e.g. it may have 8 timeslots at one time and 4 later on)" (VOCAL Technologies, 2001).

"The General Packet Radio Service (GPRS) is a new value added service that allows information to be sent and received across a mobile telephone network. It supplements today's Circuit wireless Switched Data and Short Message Service" (VOCAL Technologies, 2001). Four coding methods are available for GPRS data transmission - CS1, CS2, CS3, CS4 and are selected for use based on the prevalent environmental conditions. CS1 and CS2 offer good error detection and correction with low throughput, while CS3 and CS4 have negligible error correction or detection capabilities, but have high throughput.

Relevant GPRS features are given below

- Compliant with Layer 1 Radio, Layer 1 Vocoder,Layer 2 \& 3 ETSI GSM phase 2+.

- Table 1 shows the different data rates that can be achieved with the different coding techniques: 


\begin{tabular}{|c|c|c|c|c|}
\hline User Data Rate & CS1 & CS2 & CS3 & CS4 \\
\hline 1 Timeslot & $9.05 \mathrm{kbps}$ & $13.4 \mathrm{kbps}$ & $15.6 \mathrm{kbps}$ & $21.4 \mathrm{kbps}$ \\
\hline 8 Timeslot & $72.4 \mathrm{kbps}$ & 107.2 & 124.8 & $171.2 \mathrm{kbps}$ \\
& & $\mathrm{kbps}$ & $\mathrm{kbps}$ & \\
\hline
\end{tabular}

Table 1: Data rates for GPRS (VOCAL Technologies, 2001)

- Because it is packet switched traffic, new protocols and channel types are required. The mobile device is permanently attached to the network, providing a virtual connection without cost and data transfer (Attach). Attach procedure is executed whenever an enabled mobile station is switched on and needs to inform the network about its presence. (VOCAL Technologies, 2001)

- The two peers of a attach procedure are the mobile station (MS) and the Serving Support Node (SGSN). Depending on the availability of the packet Common Control Channel $(\mathrm{PCCCH})$, the Attach procedure may be executed on Circuit Common Control Channel (CCCCH). (VOCAL Technologies, 2001)

- Each wireless service provider's pricing plan for GPRS differs. Generally, providers offer a set amount of data for a certain fee. For example, a carrier might offer 3MB (Megabytes) of data transmission per month for $\$ 15.99$, and charge an additional $\$ .05$ per KB (Kilobyte) for anything over 3MB ("How much does a GPRS service plan cost?" n.d.).

\section{Overview of Bluetooth}

Bluetooth is a great protocol for wireless communication, with several advantages such as high data transfer rate (over 1MBps and up to 2.1 Mbps for enhanced Bluetooth channels), low implementation cost, automatic connection low interference, while consuming only 1 percent of the power consumed by conventional Wi-Fi (Wireless communication technology). The Bluetooth protocol can be used just like any other protocol such as HTTP (HyperText Transfer Protocol), FTP (File Transfer Protocol), SMTP (Simple Mail Transfer Protocol), with signal area of coverage of $100 \mathrm{~m}$ for class 1 USB which can be extended up to $500 \mathrm{~m}$ (using a $5 \mathrm{dbi} 2.4 \mathrm{GHz}$ antenna). Bluetooth operates in the $2.4 \mathrm{GHz}$ (GigaHertz) band for wireless communication.

"In order for Bluetooth devices to communicate properly, they all need to conform to the Bluetooth specification. The Bluetooth specification, like any other specification, defines the standard that a Bluetooth device should adhere to, as well as rules that need to be enforced when communicating. The Bluetooth protocol stack and profiles together comprise the Bluetooth specification" (Hopkins, 2006). Currently, mainboard chipsets for most laptop computers are equipped with Bluetooth technology. "The Bluetooth radio utilizes a signaling technique called Frequency Hopping Spread Spectrum (FHSS). The radio band is divided into 79 sub-channels. The Bluetooth radio uses one of these frequency channels at a given time. The radio jumps from channel to channel spending 625 microseconds on each channel. Hence, there are 1600 frequency hops per second. Frequency hopping is used to reduce interference caused by nearby Bluetooth devices and other devices using the same frequency band. Adaptive Frequency Hopping (AFH) is introduced in the Bluetooth 1.2 specification and is useful when a device communicates through both Bluetooth and Wi-Fi simultaneously (e.g. a laptop computer with both Bluetooth and Wi-Fi equipment). The frequency hopping algorithm can then avoid using Bluetooth channels overlapping the Wi-Fi channel in use, hence avoiding interference between your own radio communica- 
tions" (Klingsheim 2007). A Bluetooth network may consist on a maximum of eight interconnected devices to form a Piconet.

GPRS has a standard data rate of $172 \mathrm{kbps}$ maximum for data transfer and involves significant setup and configuration resources. These characteristics of GPRS and the above mentioned advantages of the Bluetooth standard therefore make Bluetooth the communication standard of choice when implementing service provisioning to mobile devices in a localized setting.

\section{Brief Review of an Existing System}

Considered in this research study, is the "Remote Control by Phone" RCP (Basta, Boubes, Jaballah, \& Al Saoudi, 2006). The RCP describes the design of a system that makes uses of an application running on a mobile device to control a PC connected to it via Bluetooth. After necessary authentication, authorization, the mobile device (here PDA's and Smart phones), runs an application that provides the following services:

\section{- Mouse Mode:}

"The mouse mode enables the user to perform basic mouse motion, right and left clicks control; moreover, RCP's mouse mode has the pageup and pagedown keys to make navigating through the desktop or any document easier."

\section{- Keyboard Mode:}

The keyboard mode types capital and small letters, it can also type numbers, in addition to that, the keyboard mode has 29 symbols. The keyboard mode also has the F5, Esc, and Caps Lock keys; the presence of these keys is very useful especially for applications like Microsoft Power Point.

\section{- Shortcuts Mode:}

This mode assigns keys on the phone's keypad to applications available on the server side, so as to make the process of running applications easier by only a click of a button. Users can assign up to 10 different applications to the phones key pad.

(Basta et al, 2006).

"Services of the RCP are limited to achieving control of the standard input peripherals (mouse and keyboard) of a PC and it is efficient in applications such as PowerPoint Presentation." (Basta et al., 2006)

While the BTServ System uses similar lower level architecture in the design of the server and client side, it essentially has different service and functionality features. BTServ is intended for implementation in corporate houses and it provides enterprise services which are consumed by multiple client devices. 


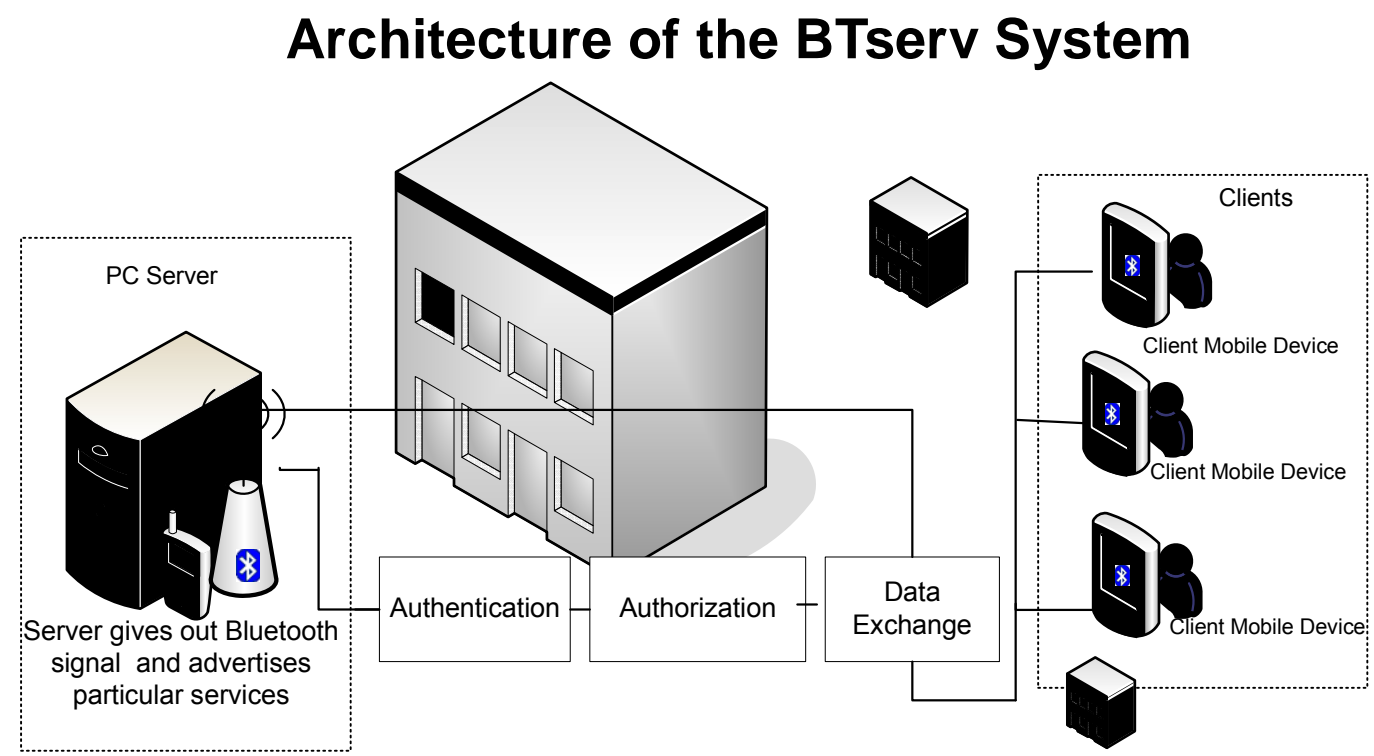

Figure 1. Schematic Diagram of the BTServ System.

Figure 1 shows the client server architecture of the BTServ system comprising two major parts: the PC Server which provides the services and the mobile devices which are the client. J2SE (Java 2 Standard Edition) has been selected for developing server side software and J2ME (Java 2 Micro Edition) for the client software, connected via Bluetooth.

Services provided to the mobile clients include:

$\begin{array}{ll}\text { - } & \text { Mail } \\ \text { - } & \text { Instant messaging } \\ \text { - } & \text { Presence Information } \\ \text { - } & \text { News } \\ \text { - } & \text { Data/File Exchange } \\ & \text { Utilities }\end{array}$

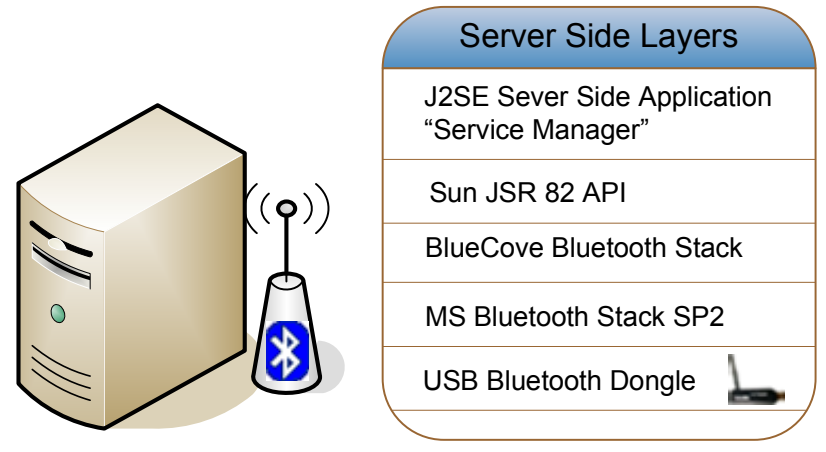

Figure 2. Layers of the Server

(Adapted from Basta et al., 2006)

The server side is divided into many layers stacked, one on the other. Each layer receives data from the one beneath it, processes it, and delivers the result to the layer on top of it. This design 
adheres to the component based design which provides a high level of coherence and cohesion. These two measures are highly preferred in the contemporary software engineering trends.

The bottom layer as displayed in Figure 2 is the Bluetooth hardware; on top of it is Microsoft's XP2 Bluetooth Enumerator and Radio (a component of the Microsoft Windows, Service Pack 2 ), which fetches the data received from the hardware layer, USB Bluetooth Dongle, and delivers this data to BlueCove's Bluetooth stack after passing through the Java Native Interface (JNI) tool; this JNI tool acts as a translator between Microsoft's XP2 Bluetooth Enumerator and Radio and BlueCove's Bluetooth stack.

A Bluetooth stack is a device driver that relies on the operating system's access to Bluetooth hardware, and there are several Bluetooth stack providers available for use. The BlueCove Bluetooth stack is used here, because of its platform independence and being open source.

On top of BlueCove's Bluetooth stack is Sun's Microsystems Bluetooth API, this API is standardized as the JSR-82; this API gives programmers the ability to perform Bluetooth operations which are discovery, device management and connectivity (Basta et al., 2006).

The top layer (Service Manager) is a set of J2SE classes, each of which performs a certain task. When data, which represents commands/requests sent by the client device, reaches this layer, it gets investigated to determine what type of command it represents - mail data, IM Data, file exchange or any other service request. To send string data to clients, RFCOMM protocol (known as the virtual serial port protocol because it allows a Bluetooth device to simulate the functions of a serial port) of the Bluetooth protocol stack is used.
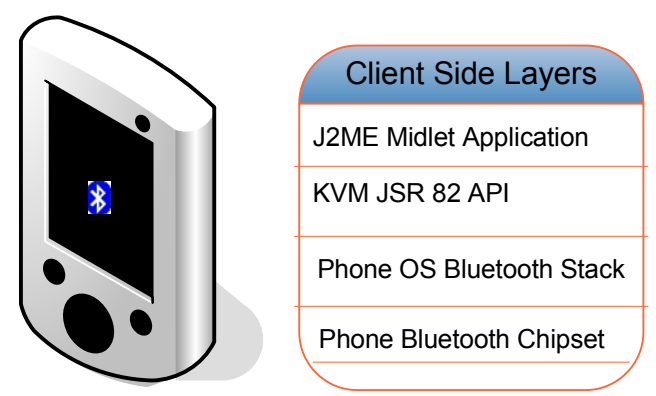

\section{Figure 3. Layers of the Client Side}

(Adapted from Basta et al., 2006)

The client operating system, which is in many cases Symbian, for smart phones or Windows CE for PDAs, has built in Bluetooth libraries that are available to programmers. The client side uses these libraries to perform Bluetooth operations. Figure 3 shows the various layers of communication on the client device. The J2ME MIDlet application communicates with the KVM (K Virtual Machine) JSR 82 API, the phone OS Bluetooth stack, Bluetooth chipset. The lower three layers are inbuilt by device manufacturers. 


\section{The BTServ System Components}

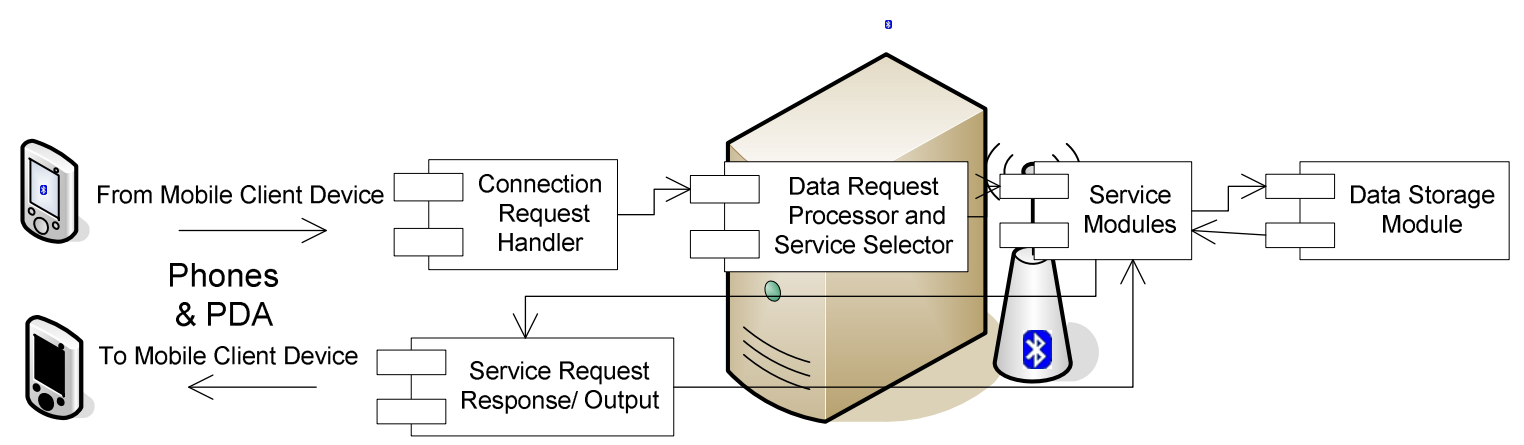

Figure 4. Component Diagram of J2SE Application on Server

Figure 4 shows the software architecture of the "Service Manager" Software running on the PC Server that essentially provides the services. It consists of components made up of software classes written in J2SE (Java 2 Standard Edition) explained below:

i. Connection Request Handler:

Contains class that receives connections, authenticates passkey for communication channel initialization.

ii. Data Request Processor and Module Selector:

Classes here analyses tags/header of data sent by clients and selects which of the service modules to direct the request to.

iii. Service Module:

Suite of classes that carry out actual Email, instant messaging, file transfer, data backup , presence information and logging services.

\section{iv. Data Storage Module:}

The classes here are involved in ensuring data persistence, by using a DBMS (Database Management System - here MSSQL is used) and data files to store data on the server. Data such as registered device name and ID, mail, News, Instant Messaging data are stored here. 


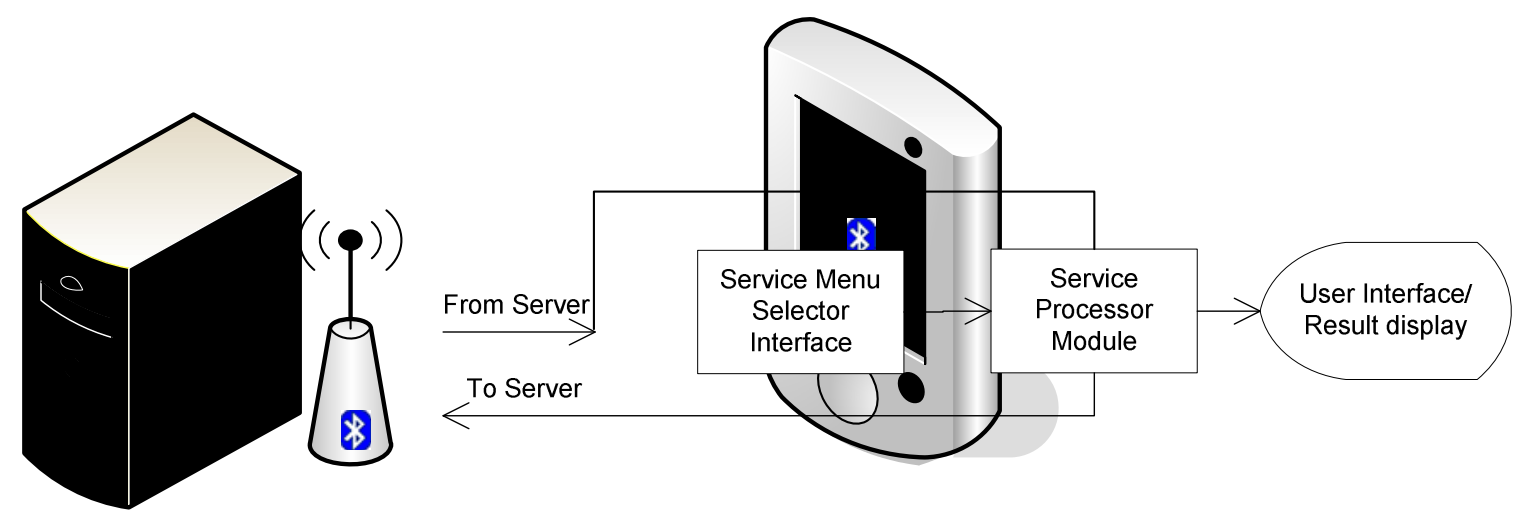

Figure 5. Component Diagram of J2ME Application on Client

The client software is a J2ME (Java 2 Micro Micro Edition ) MIDlet application, that implements the MIDP (Mobile Information Device Profile) 2.0 profile and CLDC (Connected Limited Device Configuration) 1.0 Configuration.

A MIDlet is a Java application designed to be run on a mobile device. More specifically, a MIDlet has as its core Java classes the CLDC and MIDP (Muchow, 2001).

Figure 5 shows the components of the Client software, and their functions are described below:

i. User Interface/Display: This provides the mobile device user with an interface to carry out service request .Designed using J2ME Polish, it also displays request results, such as news, mail data, etc.

ii. Service Menu Selector: This interface actively displays the user service menu.

iii. Service Processor Module: This handles communication with the server. Each data stream received by the client is read by this module, and from data header information, it determines data type i.e. mail, IM (Instant Messaging), News, and selects the right interface.

\section{Mode of Operation}

After installation of the server and client side, software, at first run the client searches for server (using Universal Unique Identifier - UUID, and services offered) and sends a connection request. Server then checks if the device has been listed for connection in its internal database and grants access to services or starts the device registration process. After device registration, services can now be accessed. Subsequent connections to the services provided by the server will be authenticated and authorized using device registration data entered on first run.

Several considerations have been made concerning security of the system.

- Passkey for communication channel initialization is handled on the software layer and not by the user.

- Only a client device that has the service ID of the services provided by the server can connect to and use those services. In this case only mobile devices that have the client software running on them. 
Presently, using servers with Bluetooth 2.0 EDR+ (Enhanced Data Rate), 7 client devices can communicate simultaneously with an instance of the Bluetooth Server Service Manager.

\section{Significance and Justification}

Service provision to mobile devices will allow for:

- Maximum and efficient use of the computing resources made available by mobile devices, enabling users to conveniently "stay connected on the fly".

- Non Dependence on GPRS Network providers, for supplying services to intranet users on the mobile platform, thus achieving a low cost intranet in which mobile devices (PDAs and Smart phones) are the intranet clients.

- Reduced cost of service provision, as GPRS costs are totally cut off. Bluetooth connections are easy to implement, common and low cost. A 500m Bluetooth dongle cost about \$30 USD

- Standard data exchange rates attainable independent $t$ of third parties such as network providers.

- Higher dependability and reliability of the Bluetooth service provisioning system, because security measures can be implemented at various levels, since all parts of the system not depend on external parties like network providers.

- An extendable system in which more services can run on the proposed framework. Various company specific applications can be integrated, to provide services to mobile users.

- Addition of economic value and increased business potential to the standard Smart phone/PDA.

\section{Conclusion}

With the implementation of this system, corporate houses can achieve better and more predictable service provision to their mobile users, incur less cost, have full control over system stability, and implement security better. However, as regards ubiquity, this system is best deployed within coverage area of Bluetooth server signals - a localized setting. Deploying it across a large area is not within the scope of this paper.

Intended future work on this research is given below.

- Considering the limitations of this system, (low area of coverage, limitation of maximum number of active connections), work in underway, in developing a grid architecture, in which several PC Servers will function in unison and provide services to a wider area of coverage and a larger number of client mobile devices. Developing an efficient means of communication between the devices, the PC Servers and clients is being concentrated on presently.

- Expand the horizon of services that can be provided through this architecture, to mobile users. The Bluetooth standard is continually undergoing evolution and presently set to harness the speed of 802.11 conventional wireless, thus event applications that require a faster data rate can be incoporated ("Bluetooth Technology," 2008)

- Make provision for modular extensions which will allow easy integration of with Enterprise Services (e.g. ERP packages) into the BTServ system. The BTServ framework 


\section{References}

Basta, M, Boubes, A. Y., Jaballah, M., \& Al Saoudi, A. (2006). Remote control by phone. Retrieved July 22, 2007 from http://www.ameinfo.com/pdfdocs/91041.pdf

Bluetooth Technology to Harness the Speed of 802.11. (2008). Retrieved February 20, 2008 from http://www.bluetooth.com/Bluetooth/Press/SIG/BLUETOOTH_TECHNOLOGY TO HARNESS TH E_SPEED OF 80211.htm

Hopkins, B. (2006). Bluetooth for Java. Retrieved July 28, 2007 from www.javabluetooth.com

How much does a GPRS service plan cost? (n.d.). Palm, Inc. Article \#26473. Retrieved February 26, 2008, from http://kb.palmone.com/SRVS/CGIBIN/WEBCGI.EXE?New,Kb=PalmSupportKB,ts=Palm_External2001,case=obj(26473)

Java Bluetooth Discussions and Tutorials. (2006). Retrieved July 14, 2007 from http://www.jsr82.com

Klingsheim, A. N. (2004). Bluetooth Programming, Master's Thesis, Department of informatics, University of Bergen, 30th June 2004. Retrieved June 8, 2007 from http://www.nowires.org, http://wireless.klings.org

Mobile Computing. (2007). Retrieved July 28, 2007 from http://en.wikipedia.org

Muchow, J. C. (2001). Core J2ME technology and MIDP. Sun Microsystems Press.

TDMA. (2006). SearchNetworking.com Definitions. Retrieved January 30, 2008 from http://searchnetworking.techtarget.com/sDefinition/0, sid7_gci214175,00.html

VOCAL Technologies. (2001). General packet radio service. Retrieved February 10,2008 from http://www.vocal.com/data_sheets/full/gprs.doc

\section{Biographies}

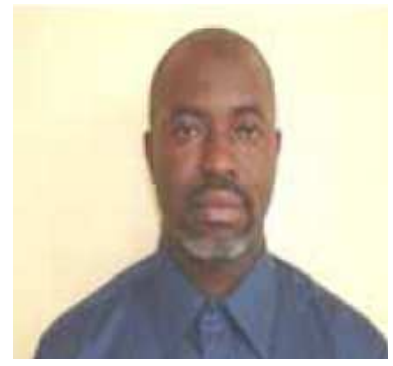

Awodele Oludele is presently a lecturer in the Department of Computer Science and Mathematics, Babcock University, Ilishan-remo, Ogun state, Nigeria. He had his M.Sc in computer science, 2002 at the University of Agriculture Abeokuta, PGD in Computer Science and BSc Mathematics both at the University of Ilorin 1998 and 1995 respectively. He is currently on his $\mathrm{Ph} . \mathrm{D}$ programme in computer science in the University of Agriculture Abeokuta. His research areas are Software Engineering, Data Communication and artificial intelligence.

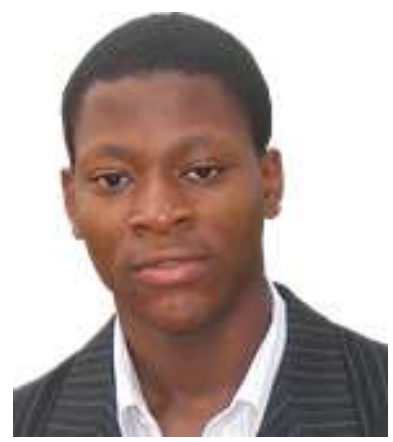

Victor .C. Dibia is a final year student researcher of Computer Science (BSc ) at Babcock University, Ilishan Remo Ogun State. Nigeria. He is an IEEE student member (Member No.80124140), ISACA member (Member No. 370098) and is Comptia Network + Certified (2007). His areas of interest are Software Development, Networks and Information System's Security and Control. 


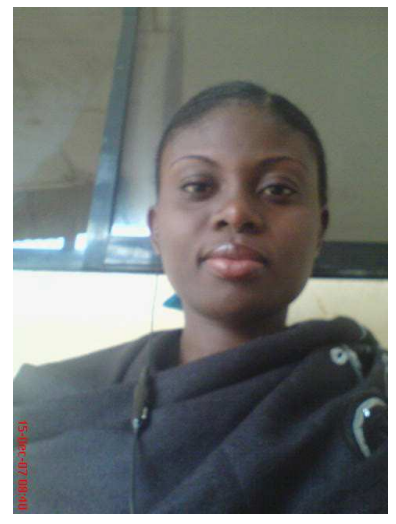

Oghenerukevwe E.L. Onoruvie is presently an undergraduate of computer technology in the Department of Computer Science and Mathematics, Babcock University, Ilishan-Remo, Ogun state, Nigeria. She hopes to graduate June $1^{\text {st }}, 2008$ and have her master's degree (M. Sc) in Information Technology after school. Her area of interest is networking administration, circuit design and analysis. She is a member of IEEE, she is Network + certified.

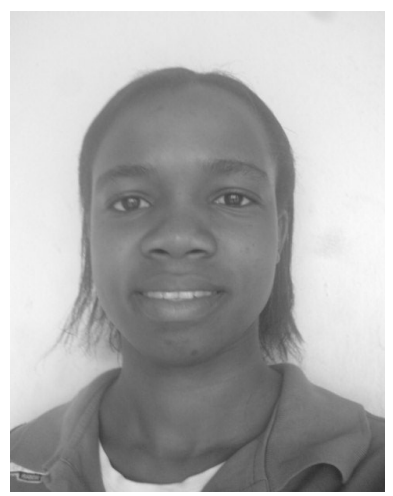

Sharon .O. Okoruwa is an undergraduate presently in Babcock University, Ilishan-Remo, Ogun State, Nigeria. She is studying Computer Technology and she is in her final year. Her area of interest is Hardware and Intelligent Agents. 